\title{
Inverse approach for the average convection coefficient induced by a forced fluid flow over an annular fin of Rectangular profile using tip temperature measurements
}

\author{
ANTONIO CAMPO \\ Department of Mechanical Engineering \\ The University of Texas at San Antonio \\ San Antonio, TX 78249, USA
}

\begin{abstract}
The objective of the present paper is to develop a simple algebraic computational procedure for the estimation of the average convection coefficient of a forced fluid flow over an annular fin of rectangular profile within the platform of inverse heat conduction problems. The data required is the tip temperatures of an annular fin of rectangular profile, which are measured in an experimental setup. Based on nonlinear regression analysis, an empirical correlation equation is constructed for the dimensionless average tip temperature depending upon the dimensionless thermo-geometrical parameter and the radius ratio. When compared against the outcome of a direct heat conduction problem, the good quality of the estimated average convection coefficient for the annular fin of rectangular profile demonstrates the feasibility of the simple algebraic computational procedure.
\end{abstract}

Keywords: annular fin of rectangular profile; inverse heat conduction problems; tip temperature measurements; empirical correlation equation for the average convection coefficient.

Received: March 26, 2021. Revised: June 30, 2021. Accepted: July 10, 2021. Published: July 16, 2021.

\section{Nomenclature}

\begin{tabular}{|c|c|}
\hline$A$ & surface area \\
\hline$A R$ & aspect ratio (length $\div$ half-thickness), $\frac{L}{t}$ \\
\hline$B i_{t}$ & transverse Biot number, $\frac{\bar{h} t}{k}$ \\
\hline$h$ & local convection coefficient \\
\hline $\bar{h}$ & average convection coefficient \\
\hline$I_{v}(X)$ & modified Bessel function of first kind and order $v=0,1$ \\
\hline$K_{v}(X)$ & $\begin{array}{l}\text { thermal conductivity } \\
\text { modified Bessel function of second kind and order } v=0,1\end{array}$ \\
\hline$L$ & length, $r_{2}-r_{1}$ \\
\hline$m$ & thermo-geometrical parameter, $\sqrt{\frac{\bar{h}}{k t}}$ \\
\hline$m L$ & dimensionless thermo-geometrical parameter, $\sqrt{\frac{\bar{h}}{k t}} L$ \\
\hline$Q$ & heat transfer rate \\
\hline$r$ & radial coordinate \\
\hline & internal radius \\
\hline
\end{tabular}




$\begin{array}{ll}r_{2} & \text { external radius } \\ t & \text { half-thickness, } \frac{\delta}{2} \\ T & \text { temperature } \\ z & \text { transversal coordinate } \\ \text { Greek symbols } & \\ \delta & \text { thickness, } 2 t \\ \eta & \text { fin efficiency } \\ \theta & \text { dimensionless temperature, } \frac{T-T_{f}}{T_{b}-T_{f}} \\ & \\ \text { Subscripts } \\ b & \text { base } \\ f & \text { fluid } \\ m & \text { measurement } \\ t & \text { tip }\end{array}$

\section{Introduction}

Direct heat conduction theory deals with the determination of the temperature distributions in solid bodies with knowledge of the initial condition, boundary conditions, heat sources and thermo-physical properties, like the thermal conductivity $k$ of the solid and the average convection coefficient $\bar{h}$ (Arpaci [1], Poulikakos [2], Yener and Kakaç [3]).

Bergles [4] has advocated that the most important passive scheme for heat transfer enhancement from hot solid bodies to nearby cold fluids consists in the incremental surface area of the solid body by attaching solid strips, called extended surfaces or fins. A variety of geometric profiles can be morphed in the form of straight fins and annular fins (Kraus et al. [5]).

Focusing on the annular fin of rectangular profile, this fin is used in numerous industrial applications, such as engines, compressors, turbines, heat exchangers, electrical equipment, electronic components, etc. [5]. The monotonic temperature descend in an annular fin of rectangular profile obeys a quasi one- dimensional heat conduction equation, i.e., an ordinary differential equation of second order with variable coefficients and nonhomogeneous, which is named the modified Bessel equation of zero order [1-3]. There are two essential quantities that affect directly the modified Bessel equation: 1) the thermal conductivity of the solid $k$ and 2) the average convection coefficient $\bar{h}$ of the forced fluid flow. Traditionally, the magnitude of $\bar{h}$ emerges from the numerical integration of the experimentally measured local convection coefficients $h$ varying with the radial and angular directions $r, \varphi$, namely

$$
\bar{h}=\frac{1}{A} \int_{A} h(r, \varphi) d A
$$

where $A$ is the surface area of the annular fin of rectangular profile.

Relying on experimental measurements, Krückels and Kottke [6] constructed contour plots of the local convection coefficient $h(r, \varphi)$ of forced air flows over the surface of an annular fin of rectangular profile. These authors demonstrated that $h$ increases from the base of the annular fin to twice or trice at the edge of the fin (see Figure 1 taken from Hausen [7]). They remarked that the radial variability of $h$ is a direct response to a thick 
boundary layer at the base compared to a thin boundary layer at the edge.

Inverse heat conduction theory deals with the estimation of the initial condition, boundary conditions, thermophysical properties, heat sources, etc. knowing the temperatures at certain internal locations in a solid body. Several exact analytical and approximate numerical techniques are recounted by Beck et al. [8], $\mathrm{O}^{\circ}$ is, $\mathrm{k}$ and Orlande [9].

The primary objective of the technical note is to develop a simple algebraic computational procedure for the estimation of the average convection coefficient $\bar{h}$ of a fluid flow over an annular fin of rectangular profile by measuring the tip temperatures at three strategic angular locations: 1) at the stagnation point $\varphi=0^{\circ}$, 2) at $\varphi=90^{\circ}$ from the stagnation point and 3) at $\varphi=180^{\circ}$ from the stagnation point. Actually, the idea behind the computational procedure is to circumvent the manipulation of the intricate measurements of the local convection coefficient distributions in two dimensions $h(r, \varphi)$.

\section{Formulation of the physical problem}

Figure 2 sketches an annular fin of rectangular profile described by three dimensions: internal radius $r_{1}$, external radius $r_{2}$ and thickness $\delta=2 t$, so that the length is $L=r_{2}-r_{1}$.

The characterization of annular fins of rectangular profile consists in two parameters: 1) the radius ratio $\frac{r_{2}}{r_{1}}$ is a geometric parameter and 2) the transverse Biot number $B i_{t}=\frac{\bar{\square} t}{k} \quad$ is a thermo-geometric parameter. In reference to the latter, $\bar{\square}$ is the average convection coefficient of the forced fluid flow over the annular fin, $t$ is the half-thickness of the annular fin and $k$ is the thermal conductivity of the solid.

A literature search reveals that the most popular procedures for the experimental measurement of the average convection coefficient $\bar{h}$ are thermocouples, infrared (IR) thermometry, thermo-chromic liquid crystals documented by Han and Wright [10], as well as naphthalene sublimation technique explained by Souza Mendes [11]).

From heat conduction theory [3-5], the temperature in straight and annular fins descends in the axial direction from the base temperature $T_{b}$ toward the fluid temperature $T_{f}$, whereas the temperature is practically uniform in the cross-section of those fins. An in-depth analysis of annular fins of rectangular profile was developed by Lau and Tan [12]. Using the finite difference method, the authors compared the quasi one- and two-dimensional heat transfer rates over a wide range of geometrical and thermal conditions. They concluded that the efficacy of the quasi one-dimensional heat conduction equation carries two major components: 1) the aspect ratio (length to half-thickness) $A r=\frac{L}{t}$ is of the order of 10 or greater and 2) the transverse Biot number satisfies the inequality $B i_{t}=\frac{\bar{h} t}{k} \leq 1$.

Under the assumptions of nearly constant thermal conductivity of the solid $k$ and average convection coefficient $\bar{\square}$ of the forced fluid flow, the quasi one-dimensional heat conduction equation in cylindrical coordinates found in Arpaci [3] is:

$$
\begin{gathered}
r^{2} \frac{d^{2} T}{d r^{2}}+r \frac{d T}{d r}-m^{2} r^{2}\left(T-T_{f}\right)=0 \\
\text { in } r_{1} \leq r \leq r_{2}
\end{gathered}
$$

where $T_{f}$ is the fluid temperature and $m=$ $\sqrt{\frac{\bar{\sigma}}{k t}}$ denotes the thermo-geometrical parameter. The thermal conductivity $k$ of solids is usually tabulated and graphed in 
compendiums, while the average convection coefficient $\bar{h}$ must be measured experimentally in a case-to-case basis.

From the standpoint of thermo-fluid experimentation, Moffat [13] has pointed out that the measurements of the average convection coefficient $\bar{h}$ in forced fluid flows over solid bodies normally withhold uncertainties that range between $\pm 10 \%$ and $\pm 20 \%$.

The boundary conditions assigned to eq. (1) are: prescribed temperature $T_{b}$ at the fin base $r_{1}$ (internal radius of the tube)

$$
T\left(r_{1}\right)=T_{b}
$$

and negligible heat loss at the fin tip $r_{2}$ (external radius)

$$
\frac{d T\left(r_{2}\right)}{d r} \approx 0
$$

At this point, it is convenient to switch from the dimensional temperature $T$ to the dimensionless temperature difference $\theta=$ $\frac{T-T_{f}}{T_{b}-T_{f}} \quad$ while retaining the radius $r$ dimensional. Correspondingly, eq. (1) transforms into the modified Bessel equation of zero order (Zwillinger [14]):

$$
\begin{aligned}
& r^{2} \frac{d^{2} \theta}{d r^{2}}+r \frac{d \theta}{d r}-m^{2} r^{2} \theta=0 \quad \text { in } \\
& r_{1} \leq r \leq r_{2}
\end{aligned}
$$

In the same way, the boundary conditions in eqs. (2a) and (2b) are rewritten as

(4a)

$$
\theta\left(r_{1}\right)=1
$$

and

$$
\frac{d \theta\left(r_{2}\right)}{d r} \approx 0
$$

\section{Exact, analytical method}

From a historical perspective, the exact, analytical solution of eq. (3) subject to eqs. (4a) and (4b) was determined originally by Harper and Brown [15] in USA and by Schmidt [16] in Germany. These efforts gave way to the exact, analytical temperature distribution in the annular fin of rectangular profile (Arpaci [1]):

$$
\theta(r)=\frac{T(r)-T_{f}}{T_{b}-T_{f}}=\frac{I_{0}(m r) K_{1}\left(m r_{2}\right)+K_{0}(m r) I_{1}\left(m r_{2}\right)}{I_{0}\left(m r_{1}\right) K_{1}\left(m r_{2}\right)+K_{0}\left(m r_{1}\right) I_{1}\left(m r_{2}\right)}
$$

where $I_{0}(*)$ and $I_{1}\left(^{*}\right)$ are the modified Bessel function of first kind and order 0 and 1 and $K_{0}(*)$, whereas $K_{1}\left(^{*}\right)$ are the modified Bessel function of second kind and order 0 and 1 (Watson [17]).

\subsection{Tip temperatures}

The exact, analytical tip temperature $T_{t}$ in the annular fin of rectangular profile comes from the evaluation of eq. (5) at the exterior radius $r=r_{2}$. That is,

$$
\begin{gathered}
\theta_{t}=\frac{T_{t}-T_{f}}{T_{b}-T_{f}}= \\
\frac{I_{0}\left(m r_{2}\right) K_{1}\left(m r_{2}\right)+K_{0}\left(m r_{2}\right) I_{1}\left(m r_{2}\right)}{I_{0}\left(m r_{1}\right) K_{1}\left(m r_{2}\right)+K_{0}\left(m r_{1}\right) I_{1}\left(m r_{2}\right)}
\end{gathered}
$$

where $T_{t}=T\left(r_{2}\right)$ stands for the tip temperature. Due to the intricate structure of eq. (6), the numerical evaluation of $T_{t}$ is extremely elaborate and time consuming even with modern algebraic symbolic codes, like Mathematica, Maple and Matlab (von Zur Gathen and Gerhard [18]). 


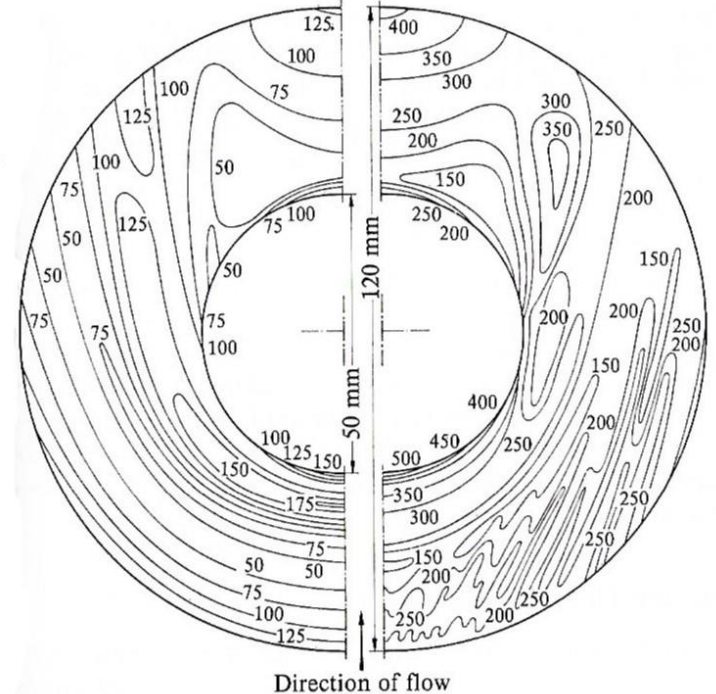

Figure 1. Experimental-based contour plots for the local convection coefficient $h$ over an annular fin of rectangular profile with inner diameter $D_{1}=50 \mathrm{~mm}$ and outer diameter $D_{2}=$ $120 \mathrm{~mm}$ made by Krückels and Kottke [6]. The air flow velocity on the left part is $2 \mathrm{~m} / \mathrm{s}$ and on the right part is $10 \mathrm{~m} / \mathrm{s}$. The units of $h$ are expressed in $\mathrm{Kcal} /\left(\mathrm{m}^{2}-\mathrm{h}-\mathrm{K}\right)$, namely 16.3 higher in $\mathrm{W} /\left(\mathrm{m}^{2}-\mathrm{K}\right)$. The figure is taken from Hausen [7].

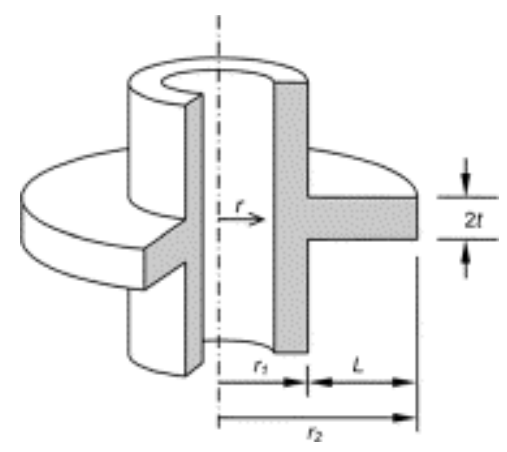

Figure 2. Sketch of an annular fin of rectangular profile with length $L$ and thickness $\delta=2 t$



Figure 3. Location for the tip temperature measurements in an annular fin of rectangular profile exposed to a forced fluid flow

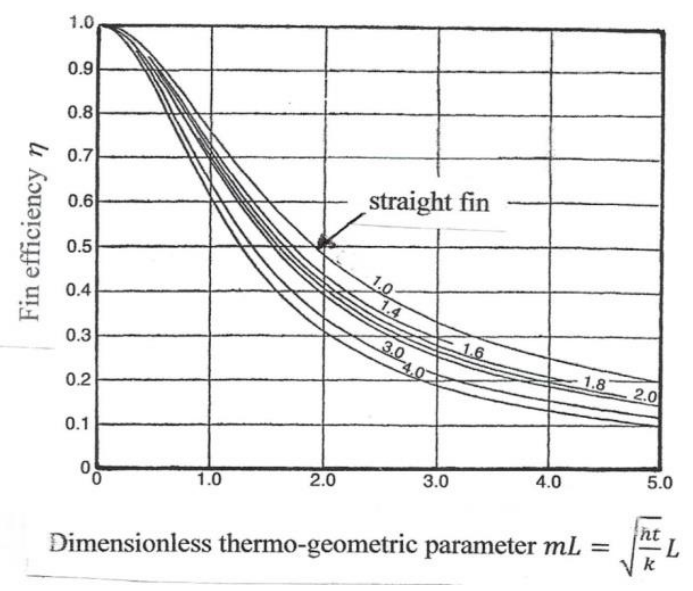

Figure 4. Fin efficiency diagram due to Gardner [20]. 


\section{Experimental measurement of the tip temperatures}

As already mentioned, the central idea for undertaking the present study is to estimate the average convection coefficient $\bar{h}$ of a forced fluid flow over an annular fin of rectangular profile using measurements of the tip temperature along the periphery of the fin. The strategic locations are indicated in Figure 3: 1) the stagnation point $\varphi=0^{\circ}$, 2) $\varphi=90^{\circ}$ from the stagnation point and 3 ) $\varphi=180^{\circ}$ from the stagnation point. Because of symmetry, the tip temperature at $\varphi=270^{\circ}$ from the stagnation point being equal to the tip temperature at $\varphi=$ $90^{\circ}$ from the stagnation point is not needed. The three measured tip temperature set forth the average measured tip temperature

$$
\left.T_{t, m, \frac{3 \pi}{2}}\right)
$$

$$
\bar{T}_{t, m}=\frac{1}{3}\left(T_{t, m, 0}+T_{t, m, \frac{\pi}{2}}+\right.
$$

so that the dimensionless average measured tip temperature is

$$
\bar{\theta}_{t, m}=\frac{\bar{T}_{t, m}-T_{f}}{T_{b}-T_{f}} .
$$

Having secured the magnitude $\bar{\theta}_{t, m}$, the next step requires the calculation of the thermo-geometrical parameter $m$ in the arguments of the six modified Bessel functions. Thereby, from eq. (6) the transcendental equation that results is

$$
\begin{gathered}
\bar{\theta}_{t, m}= \\
\frac{I_{0}\left(m r_{2}\right) K_{1}\left(m r_{2}\right)+K_{0}\left(m r_{2}\right) I_{1}\left(m r_{2}\right)}{I_{0}\left(m r_{1}\right) K_{1}\left(m r_{2}\right)+K_{0}\left(m r_{1}\right) I_{1}\left(m r_{2}\right)}
\end{gathered}
$$

where the internal radius $r_{1}$ and the external radius $r_{2}$ of the annular fin are known quantities and $m$ is the unknown quantity. Needless to say, eq. (8) is of strenuous convoluted form because between the numerator and the denominator it contains six modified Bessel functions. Obviously, the computation of the root $m$ is extremely difficult, even with advanced root finding techniques, such as the fixed point iterative method, the Newton-Raphson method, the bisection method, etc. (Chapra and Canale [19]). In view of the impending obstacle, an alternate procedure for computing the root $m$ in eq. (8) is de rigueur and it will be pursued in the following paragraphs.

In the case of the straight fin of rectangular profile, the dimensionless tip temperature $\theta_{t}$ is expressible with the exact, analytical equation (Arpaci [3]))

$$
\frac{1}{\theta_{t}}=\cosh m L
$$

where $m L=\sqrt{\frac{\bar{h}}{k t}} L$ is the dimensionless thermo-geometrical parameter.

In the case of the annular fin of rectangular profile, the fin efficiency diagram devised by Gardner [20] conforms to the graphical representation of the twovalued function of the fin efficiency $\eta=$ $f\left(m L, \frac{r_{2}}{r_{1}}\right)$. The function $f(*)$ is articulated with $m$ inside the closed interval $0 \leq m L \leq 5$ on the abscissa and the family of curves represented by the radius ratio $\frac{r_{2}}{r_{1}}$ inside the closed interval $1 \leq \frac{r_{2}}{r_{1}} \leq 5$ as seen in Figure 4.

Inspired in the compact form of eq. (9), we applied nonlinear regression analysis to the collected data $\bar{\theta}_{t}, m L, \frac{r_{2}}{r_{1}}$ forming eq. (8), which obviously is restricted to the closed intervals $0 \leq m L \leq 5$ and $1 \leq \frac{r_{2}}{r_{1}} \leq$ 5. The goal now is to construct an empirical correlation equation for $\bar{\theta}_{t}$ engaging two independent variables, one is the hyperbolic cosine of $m L$ and the other is the radius ratio $\frac{r_{2}}{r_{1}}$. Thereby, the code Table Curve 3D 4.0 
[21] delivers the empirical correlation equation

$$
\frac{1}{\bar{\theta}_{t}}=0.5\left[\left(1-\sqrt{\frac{r_{2}}{r_{1}}}\right)+\left(1+\sqrt{\frac{r_{2}}{r_{1}}}\right) \cosh m L\right]
$$

owning a high correlation coefficient $\mathrm{R}-$ square $=0.9986$. As expected, eq. (10) collapses into eq. (9) linked to the straight fin of rectangular profile identified by the limiting radius ratio $\frac{r_{2}}{r_{1}}=1$.

Isolating the thermo-geometrical parameter $m$ in the argument of the hyperbolic cosine in eq. (10), the explicit algebraic equation that turns out is:

$$
m=\frac{1}{L} \cosh ^{-1}\left(\frac{\frac{2}{\bar{\theta}_{t}}+\sqrt{\frac{r_{2}}{r_{1}}}-1}{\sqrt{\frac{r_{2}}{r_{1}}}+1}\right)
$$

(11)

Note that this equation contains four independent variables; three independent variables are the internal radius ratio $r_{1}$, the external radius $r_{2}$ and the length $L$, whereas the other independent variable is the measured dimensionless average tip temperature $\bar{\theta}_{t}$ coming from eq. (7a).

In retrospect, it is quite obvious that manipulation of the compact explicit form of $m$ in eq. (11) involving the inverse of the hyperbolic function $\cosh ^{-1}(*)$ is much simpler than manipulation of the root $m$ in the tangled implicit eq. (8) containing six modified

Bessel

\section{functions}

$$
I_{0}\left(m r_{1}\right), K_{0}\left(m r_{1}\right), I_{0}\left(m r_{2}\right), K_{0}\left(m r_{2}\right) \text {, }
$$
$I_{1}\left(m r_{2}\right)$ and $K_{1}\left(m r_{2}\right)$. As a direct consequence, the evaluation of $m$ in eq. (11) is easily manageable with a pencil on a back-of-an-envelope using an inexpensive pocket-size calculator.

Once the thermo-geometrical parameter $m=\sqrt{\frac{\bar{\square}}{k t}}$ is evaluated, the estimation of the average convection coefficient $\bar{\square}$ is obtainable with the algebraic equation

$$
\bar{h}=k t m^{2}
$$

in connection to the pair of closed intervals $0 \leq m L \leq 5$ and $1 \leq \frac{r_{2}}{r_{1}} \leq 5$.

\section{Presentation of results}

Fundamentally, the estimation of the average convection coefficient $\bar{h}$ of a forced fluid flow over an annular fin of rectangular profile in this study falls under the category of an inverse heat conduction problem [8,9]. The algebraic procedure conducive to the estimation of $\bar{h}$ will be done with a "simulated tip temperature measurement" of the three tip temperatures using as data an example a direct heat conduction problem.

\subsection{Example 1.3 on pp. 29-31 of Kraus et}

al. [2]: Radial fin of rectangular profile.

A radial fin of rectangular profile has outer diameter $D_{2}=25 \mathrm{~cm}$, inner diameter $D_{1}=10 \mathrm{~cm}$ and thickness $\delta=0.25 \mathrm{~cm}$. The fin is made from a steel with thermal conductivity $k=40 \quad \mathrm{~W} /(\mathrm{m}-\mathrm{K})$. The temperature at the fin base is $T_{b}=110^{\circ} \mathrm{C}$ and the surrounding fluid has a temperature $T_{f}=$ $35^{\circ} \mathrm{C}$ with a heat transfer coefficient $\bar{\square}=40$ $\mathrm{W} /\left(\mathrm{m}^{2}-\mathrm{K}\right)$. Determine (a) the fin efficiency $\eta$, (b) the tip temperature $T_{\text {tip }}$ and (c) the heat dissipation $Q$.

1) First, the quantities taken from the solution of the example are:

(a) the fin efficiency $\eta=0.343$

(b) the tip temperature $T_{t}=48.5^{\circ} \mathrm{C}$

(c) the heat dissipation $Q=84.86 \mathrm{~W}$

2) Second, the quantities taken from the statement of the example are:

(d) the half-thickness $t=0.00125 \mathrm{~m}$

(e) the radius ratio $\frac{r_{2}}{r_{1}}=\frac{0.125}{0.05}=2.5$ 
(f) the length $L=r_{2}-r_{1}=$

$12.5 \mathrm{~cm}-5 \mathrm{~cm}=7.5 \mathrm{~cm}=0.075 \mathrm{~m}$

(g) the dimensionless tip temperature

$\theta_{t}=\frac{T_{t}-T_{f}}{T_{b}-T_{f}}=\frac{48.5-35}{110-35}=0.18$

To commence the analysis with the available information, the substitution of the geometric quantities $\frac{r_{2}}{r_{1}}=2.5, L=0.075 \mathrm{~m}$, along with the "simulated tip temperature measurement" $\theta_{t}=0.18$ into eq. (11) furnishes the thermo-geometrical parameter $m=\sqrt{\frac{\bar{h}}{k t}}=2.12 \mathrm{~m}^{-1}$. Thereupon, evaluation of eq. (12) supplies the average convection coefficient $\bar{\square}=39.95 \mathrm{~W} /\left(\mathrm{m}^{2}-\mathrm{K}\right)$ complying with the objective of this work. Actually, the relative error between the estimated $\bar{\square}$ and the given $\bar{\square}=40 \mathrm{~W} /\left(\mathrm{m}^{2}-\mathrm{K}\right)$ in the statement of the example turns out to be $0.125 \%$, i.e., a disparity that is considered legitimate in engineering practice. In fact, it should be recalled at this point that measurements of the average convection coefficient $\bar{h}$ in forced fluid flows over solid bodies withhold uncertainties that usually range between $\pm 10 \%$ and $\pm 20 \%$ (Moffat [13]).

For completeness, calculation of the heat dissipation and the fin efficiency gives $Q=$ $84.75 \mathrm{~W}$ and $\eta=0.326$. Consequently, the relative error for $Q$ amounts to $0.13 \%$ and the relative error for $\eta$ amounts to $4.96 \%$; both numbers are considered insignificant in view of the complexity of the inverse heat conduction problem under study.

\section{Conclusions}

The outcome of the theoretical study devoted to experimental thermo-fluid dynamics has demonstrated that the measurement of three tip temperatures in an annular fin of rectangular profile is sufficient for the estimation of the average convection coefficient $\bar{\square}$ over the annular fin with reasonable accuracy within the bounds of engineering practice. From a conceptual standpoint, the proposed algebraic computational procedure deals with inverse heat conduction theory. Surely, the algebraic computational procedure can be easily extended to all annular fins of curved profiles documented in the handbook on extended surface heat transfer (Kraus et al. [2]).

It is expected that instructors of heat transfer courses as well as thermal design engineers will benefit from the outcome of the present work.

\section{References}

[1] V. Arpaci, Conduction Heat Transfer, Addision-Wesley, Reading, MA, 1966.

[2] D. Poulikakos, Conduction Heat Transfer, Prentice Hall, Englewood Cliffs, NJ, 1993.

[3] Y. Yener and S. Kakaç, Heat Conduction, $4^{\text {th }}$ edition, CRC Press, Boca Raton, FL, 2008.

[4] A. E. Bergles, Techniques to Enhance Heat Transfer. In Handbook of Heat Transfer, Chapter 11, Rohsenow, W. M., Hartnett, J. P., Cho, Y. I., eds., McGrawHill, New York, NY, 1998.

[5] A. D. Kraus, A. Aziz and J. Welty, Extended Surface Heat Transfer, John Wiley and Sons, New York, NY, 2001.

[6] W. Krückels and V. Kottke, Untersuchung über die Verteilung des Wärmeübergans an Rippen und RippenrohrModellen, Chem. Ing. Tech., Vol. 42, No. 6, pp. 355-362, 1970.

[7] H. Hausen, Heat Transfer in Counterflow, Parallel Flow and Cross Flow, p. 92, McGraw-Hill, New York, NY, 1983.

[8] J. V. Beck, B. Blackwell and C. R. St. Clair, Inverse Heat Conduction: Ill-Posed Problems, John Wiley, New York, NY, 1985. 
[9] M. N. O' is, k and H. R. B. Orlande, Inverse Heat Transfer: Fundamentals and Applications, Taylor \& Francis, Boca Raton, FL, 2000.

[10] J.-C. Han and L. M. Wright, Experimental Methods in Heat Transfer and Fluid Mechanics, 1st Edition, CRC Press, Boca Raton, FL, 2020.

[11] P. R. Souza Mendes, The naphthalene sublimation technique, Experimental Thermal Fluid Science, Vol. 4, Issue 5, pp. 510-523, 1991.

[12] W. Lau and C.-W. Tan, Errors in one-dimensional heat transfer analysis in straight and annular fins, ASME Journal of Heat Transfer, Vol. 95, No. 4, pp. 549-551, 1973.

[13] R. J. Moffat, Describing the uncertainties in experimental results, Experimental Thermal Fluid Science, Vol. 1, Issue 1, pp. 3-17, 1988.

[14] D. Zwillinger, Handbook of Differential Equations, Academic Press, Orlando, FL, 1997.

[15] D. R. Harper and W. B. Brown, Mathematical Equations for Heat Conduction in the Fins of Air-Cooled Engines, NACA Report No. 158, 1922.

[16] E. Schmidt, Die Wärmeübertragung durch Rippen, Z. Verein Deutscher

Ingenieure, Vol. 70, pp. 885-889, pp. 9471128, p. 1504, 1926.

[17] G. N. Watson, A Treatise on the Theor of Bessel Functions, 2nd edition, Cambridge University Press, London, England, UK, 1966.

[18] J. von Zur Gathen and J. Gerhard, Modern Computer Algebra, 3rd edition, Cambridge University Press, Cambridge, London, England, UK, 2013.

[19] S. C. Chapra and R. P. Canale, Numerical Methods for Engineers, 7th edition, McGraw-Hill, New York, NY, 2014.
[20] K. A. Gardner, Efficiency of extended surfaces, Transactions of ASME, Vol. 67, pp. 621-631, 1945.

[21] https://systatsoftware.com

\section{APPENDIX: Two-Dimensional Heat Conduction}

The analysis of the dimensionless temperatures $\theta(r, t)$ in annular fins of rectangular profile was done by Lau and Tan [12] using the two formulations: 1) the approximate quasi one-dimensional heat conduction model given by eqs. (3), (4a) and (4b) and 2) the exact two-dimensional heat conduction model (Arpaci [1])

(6)

$$
\frac{\partial^{2} \theta}{\partial r^{2}}+\frac{1}{r} \frac{\partial \theta}{\partial r}+\frac{\partial^{2} \theta}{\partial z^{2}}=0
$$

with the imposed boundary conditions

(7a)

$$
\theta\left(r_{1}, z\right)=1
$$

$$
\frac{\partial \theta\left(r_{2}, z\right)}{\partial r} \approx 0
$$

where $B i_{t}=\frac{\bar{h} t}{k}$ is the transverse Biot number.

\section{Creative Commons Attribution License 4.0 (Attribution 4.0 International, CC BY 4.0)}

This article is published under the terms of the Creative Commons Attribution License 4.0

https://creativecommons.org/licenses/by/4.0/deed.en_US 